\title{
A Bioética no Contexto do Idoso com HIV/AIDS em exercício da sexualidade
}

\section{Bioethics in the context of the elderly living with HIVIAIDS and practicing sex}

\author{
Moema Santos Souza \\ UESB, Bahia, Brasil \\ amonsouza2@hotmail.com \\ Wanderley Matos Reis Junior \\ UESB, Bahia, Brasil \\ wreisjunior@gmail.com \\ SergioYarid \\ UESB, Bahia, Brasil \\ syarid@hotmail.com \\ Ricardo Mazzon Sachetto \\ UESB, Bahia, Brasil \\ prof.ricardomazzon@hotmail.com \\ Rita Narriman Silva de Oliveira Boery \\ UESB, Bahia, Brasil \\ rboery@gmail.com
}

Resumo: O exercício da sexualidade do idoso que convive com HIV/AIDS exige novo direcionamento, tendo em vista as transformações sociais ocorridas nas últimas décadas. Temas relacionados ao comportamento humano após os 60 anos de idade precisam ser debatidos à luz da bioética, levando-se em consideração as questões de gênero e o fenômeno da feminização na velhice. As considerações a serem apontadas mostram que ainda há dificuldades no enfrentamento de obstáculos sociais para o exercício da sexualidade, sendo agravadas quando o idoso convive com HIV/AIDS. Aspectos sociais relacionados aos papéis definidos pelo gênero, bem como influenciados pela feminização na velhice, requerem atenção da bioética na perspectiva de mudar a visão que se tem acerca da sexualidade do idoso.

Palavras chave: Envelhecimento, bioética, Síndrome da Inmunodeficiência Adquirida, idoso.

Abstract: Sexual practice among elderly living with HIV/AIDS requires new directions, considering the social changes that have occurred in recent deca- 
des. Issues related to human behavior after turning 60 years of age need to be discussed according to bioethics, taking into account gender aspects and the phenomenon of elderly's feminization. The considerations to be mentioned show that there are still difficulties to overcome social obstacles in the practice of elderly's sexuality, aggravated when they live with HIV/AIDS. Social aspects related to positions defined by gender and age feminization require attention from bioethics in view of changing the vision we have about elderly's sexuality.

Keywords: Aging, bioethics, Acquired Immunodeficiency Syndrome, elderly.

\section{Introdução}

Nos últimos anos o aumento da expectativa de vida do brasileiro tem ganhado notoriedade. O Brasil não é mais um país de jovens e a quantidade de idosos já representa uma parcela importante da população. As consequências destas alterações envolvem questões políticas, sociais e econômicas, que interferem na inter-relação entre as pessoas e o idoso, bem como na relação do idoso com o mundo.

Neste cenário, o desempenho da sexualidade do idoso tem sido influenciado pelo determinismo social quanto ao gênero nas definições de papéis, e ainda por fatos como a feminização da velhice, situações que têm sido negligenciadas em virtude da crença de que o idoso é incapaz, um ser assexuado, sem vontades, e limitado em suas sensações e prazeres.

Esta visão tem impedido o desenvolvimento de práticas comportamentais saudáveis pelos idosos e, ao mesmo tempo, favorecido o aumento na prevalência de doenças sexualmente transmissíveis (DST), com destaque para HIV/AIDS. Assim, ante a realidade do envelhecimento populacional e a escusa da sociedade em discutir a sexualidade do idoso, urge a necessidade de debater, a partir da bioética, alguns conflitos que o idoso que convive com HIV/AIDS enfrenta no exercício da sexualidade.

\section{O envelhecimento humano no contexto da epidemia da AIDS}

O crescimento demográfico da população idosa exerce importante influência sobre o desenvolvimento e funcionamento das sociedades, de forma que os países consideram as prováveis 
repercussões deste acontecimento como assunto prioritário no âmbito da saúde pública e da economia $(1,2)$.

De acordo com o Instituto Brasileiro de Geografia e Estatística (IBGE), a expectativa média de vida em países desenvolvidos varia de 79,2 anos nos Estados Unidos, a 82,7 no Japão. Na China foi de 73,0 e no Brasil a média já atingiu 73,2 em 2010 (3).

O fenômeno do crescimento do número de indivíduos acima de 60 anos no mundo e no Brasil, bem como os rápidos avanços da medicina e da tecnologia, têm favorecido as pessoas a buscarem manter um envelhecimento mais ativo e com melhor qualidade de vida, inclusive prolongando sua atividade sexual $(4,5)$. Isso pode tornar os idosos uma população mais exposta aos riscos de DST, com destaque para o HIV/AIDS $(4,6)$.

Nos últimos anos o processo de envelhecimento tem sido acompanhado por um evento negativo e inesperado, que é o aumento de pessoas acima de 60 anos infectadas com HIV/AIDS, o que tem se constituído como um dos desafios para governos, comunidade científica e profissionais de saúde, pois é um dos mais sérios problemas de saúde pública mundial $(4,5,6)$.

Estima-se que 33,4 milhões de pessoas vivem com HIV/AIDS em todo o mundo. Na América Latina, o Brasil é o país com maior número de casos (4). Os novos números da AIDS no Brasil, enquanto doença manifestada atualizados até junho de 2010, contabilizam 592.914 casos registrados desde 1980. Em relação às faixas etárias, observa-se que a maior proporção destes se encontra entre os 40 e 49 anos. Nos indivíduos a partir dos 60 anos, verifica-se um aumento importante em ambos os sexos, que passaram de 394 casos em 1999 para 938 em 2009, no sexo masculino; e no feminino, de $191 \mathrm{em}$ 1999 para 685 em 2009 (7).

Durante quase duas décadas a ocorrência de AIDS entre idosos foi um assunto ignorado. E a resistência da sociedade em admitir que pessoas com mais de 50 anos continuem tendo vida sexual ativa contribuiu para o aumento do número de infectados $(4,8)$. 


\section{A bioética no contexto do idoso com HIV/AIDS e o exercício de sua sexualidade}

O enfrentamento destas questões se apresenta como um grande desafio da bioética, pois traz em seu bojo conflitos sociais que envolvem diretamente dilemas cotidianos que carecem de atenção por parte do poder público e da sociedade. Isto, porque as novas demandas que surgem com o aumento de idosos no cenário mundial e brasileiro alteram toda estrutura social: desde a esfera familiar até o mercado de trabalho, políticas públicas e atividades sociais de modo geral, e ainda valores e concepções de vida $(9,10)$.

A partir de inquietações e discussões similares a esta é que a bioética surgiu como mediadora de conflitos e como defensora da diversidade como condição moral da humanidade. Seu nascimento foi precedido de importantes transformações ocorridas no cenário social, político e tecnológico entre as décadas de 1960 e 1970 (10).

Alguns episódios da história da humanidade podem ser caracterizados como indutores de discussões bioéticas: as duas guerras mundiais e os experimentos nazistas nos campos de concentração; os tratados internacionais (Declaração de Helsinki e a Declaração Universal dos Direitos Humanos); movimentos socioculturais ocorridos nos países ocidentais nos anos 1960 e 1970 (emancipação feminista, movimento hippie, revolução sexual); divulgação do Relatório Belmont, nos Estados Unidos, em 1978 (organizado pela Comissão Nacional para Proteção dos Seres Humanos da Pesquisa Biomédica e Comportamental) (10).

Outros eventos também são lembrados como de importância fundamental na origem da bioética, como a luta pelos direitos dos pacientes nos Estados Unidos, e a publicação do livro Princípios da Ética Biomédica, em 1979 (11), que consolidou a força teórica da bioética. A obra apresenta os princípios morais que devem ser aplicados à biomedicina e sugere quatro deles como base de uma teoria bioética consistente: Autonomia, que corresponde ao respeito pelo direito de cada pessoa de autogovernar-se, sendo protegidas de qualquer forma de abuso; beneficência, que enfatiza a necessidade de buscar sempre o bem-estar dos enfermos; não maleficência, que consiste em não lesar, em primeiro lugar; e justiça, que é o princípio 
formal de equidade, no qual os iguais devem ser tratados de modo igual e os desiguais, de modo desigual $(9,11)$.

\section{Bioética do cotidiano}

A questão de idosos portadores de HIV/AIDS é mais um evento importante e deve fazer parte das discussões bioéticas atuais, pois o interesse nas problemáticas da saúde pública tem sido legitimado pela Sociedade Brasileira de Bioética no campo acadêmico desde os anos 90 (12). Aliado a isso, a bioética e a saúde coletiva convergem epistemologicamente, levando-se em consideração a complexidade dos determinantes sociais, subjetivos, contextuais e ambientais da saúde. Este momento requer que a transdisciplinaridade, indispensável no modelo de atenção de vigilância à saúde, considere as ações humanas determinadas pela subjetividade e pelo contexto sociocultural, sabendo que este olhar é um desafio para a avaliação moral (13).

Assim, a infecção configura-se como um fenômeno social de amplas proporções, impactando princípios morais, religiosos e éticos, procedimentos de saúde pública e de comportamento privado, principalmente relativo à sexualidade, sendo esta uma das problemáticas mais evidentes.

A pessoa que convive com HIV/AIDS enfrenta todos os dias situações que a deixam em constrangimento ou conflitos difíceis de serem superados quando se identifica como tal.

Assim, sempre que possível, opta pela omissão, porque, em alguns casos, a informação é obtida através de coerção e assédio moral, reforçando o estigma que a doença tem trazido consigo ao longo dos anos e, consequentemente, afastando-a do convívio social (4,5,9,14-21).

Há muito, por preconceito e/ou falta de conhecimento, as relações sociais das pessoas que convivem com HIV/AIDS são comprometidas e limitadas em todos os seus aspectos. No imaginário social, a doença adquiriu, em seus primórdios, muitos estigmas, como o medo que algumas pessoas têm de se aproximar das pessoas infectadas, acreditando que sofrerão o contágio, ou a relação que as mesmas estabelecem com a vulnerabilidade e a pauperização $(4,5,9,14-22)$.

Os dilemas enfrentados pelos portadores do HIV/AIDS extrapolam as situações da coexistência, pois adquirem um envolvimento 
psicológico e emocional que, numa via de mão dupla, levam o indivíduo ao ostracismo social e ao comprometimento das relações sociais, representando, em última análise, a perda da identidade deste como ser humano único e plural, ao mesmo tempo, pertencente a uma sociedade e dependente da relação com o outro $(9,14,15,23)$.

Assim, o estigma da infecção se agrava quando as pessoas que vivem com HIV/AIDS são idosos. Estes, em sua maioria, não possuem doenças infectocontagiosas, mas conforme transição epidemiológica, desenvolvem doenças crônico-degenerativas e enfrentam problemas relacionados à velhice, como isolamento, esquecimento, maus-tratos, abandono, perda de direitos, preconceito, dentre outros. Agora, veem-se ante uma nova realidade cruel, a infecção pelo HIV/AIDS (4,5,9,14-21).

Contudo, as concepções que a sociedade ainda hoje mantém acerca da sexualidade dos idosos permanecem como tabu que gera obstáculos e configuram-se como problemas a serem debatidos e refletidos à luz da bioética, na perspectiva de se estabelecer um novo paradigma quanto ao enfrentamento do avanço das DST nessa faixa etária, dentre as quais, sem dúvida, a infecção por HIV/AIDS se destaca, graças à implicação das múltiplas consequências que a seguem $(9,17,18,19,21,24,25)$.

Portanto, diante das diversas situações enfrentadas pelos idosos, muitos dilemas éticos associados ao cuidado e atenção à saúde tornam-se patentes, como: de que forma a assistência à saúde tem se organizado para atender ao idoso que convive com HIV/AIDS? Como se dá a relação entre o profissional da saúde e o idoso? Este tem sido prioridade nas políticas públicas? Ele tem sido violado quanto ao seu direito no acesso ao tratamento medicamentoso? Por que os equipamentos de comunicação não dão ênfase a este público?

Entendendo que a sexualidade é uma função fisiológica que implica um comportamento ao qual se atribuem valores morais (21), a questão que emerge como dilema a partir desses pressupostos é: quais conflitos éticos o idoso que convive com HIV/AIDS enfrenta no exercício da sexualidade? Esse questionamento possui múltiplas facetas, mas aqui se atêm a duas: a de gênero e, mais especificamente, as do fenômeno da feminização na velhice.

A sexualidade possui um significado muito amplo, envolvendo sentimentos, carícias, palavras, entre outros. Inclui a atividade sexual, mas não se resume em sexo, podendo ser entendida como 
energia, libido, caracterizada por uma capacidade de relacionarse com o outro, com a vida. Esta capacidade, importante para o significado da existência humana, tem sido condicionada a uma incapacidade latente vivida pelos idosos que convivem com HIV/ AIDS $(9,18,19,24,25,26)$.

Por conseguinte, as formas como as pessoas se relacionam com a sua sexualidade dependem, além de outros fatores, da influência do meio social, da história de vida, das informações que adquiriu sobre a mesma, e da construção de sua identidade sexual, o que exerce influência na execução da própria sexualidade. Em muitos casos, isso tem sustentado a visão errônea da sexualidade na velhice pela sociedade e pelos próprios idosos, principalmente quando se descobre que estão infectados por um vírus de tratamento difícil e estigmatizante $(9,14-26)$.

Logo, é possível perceber que vários estudos revelam padrões de comportamento da sexualidade exercidos por idosos e representam modelos antigos considerados normais, como alguns papéis exercidos pelos homens, a exemplo do fato de possuírem vários relacionamentos, em detrimento às mulheres acometidas por normas morais rígidas, consideradas tradicionais $(17,18,19,22,24-26)$.

Sendo assim, o padrão de comportamento da sexualidade equivocado é um dos fatores que tem contribuído para o aumento da incidência de infecções pelo HIV/AIDS, e encontra guarida quando muitos homens idosos, mesmo em relacionamentos estáveis, procuram outros tipos de aventuras, por acreditarem que assim potencializarão sua virilidade. Já as mulheres idosas levadas a viver uma vida solitária quase sempre assumem responsabilidades sobre toda a família. Vale salientar que, quando viúvas, podem alcançar uma posição mais livre e mais pública, o que favorece a manutenção ou o incremento de várias relações afetivas e sexuais (17-19,22,24-26).

Nessa perspectiva, para que haja conscientização, deve-se considerar que não é o exercício da sexualidade dos idosos que os torna mais vulneráveis a contrair o vírus, mas a prática sexual desprotegida $(15,22)$.

Portanto, é preciso esclarecer à população que a sexualidade deve ser exercida levando-se em consideração as conquistas já obtidas durante os anos, que trouxeram mudanças importantes na 
literatura, nas artes, na legislação e nas ciências, sem perder de vista que o sexo requer precaução e racionalidade; não podendo se restringir ao ato em si, mas a várias experiências, que proporcionam um prazer incomparável à satisfação de uma necessidade fisiológica fundamental $(9,17-19,21,24,25)$.

O corpo representa para as mulheres a expressão da razão de ser para si mesma e para o outro; portanto, a longevidade e suas marcas, manifestadas pelo fenótipo corporal, reflete a ideia socialmente estereotipada das mulheres velhas, cuja função social limita-se a cuidar da família, da casa, do marido, na participação da criação dos netos, buscando ser socialmente aceita, pois ainda são muito discriminadas quando buscam o exercício da sexualidade, vivenciando um tratamento desigual frente aos homens (17-19, 22, 24-26).

Ao contrário do que se pensa, as mulheres possuem menores dificuldades quanto ao desenvolvimento da vida sexual na velhice, pois a forma de lidar com suas relações afetivas sempre esteve relacionada a sensações corporais, normalmente exercitando a sexualidade de corpo inteiro $(9,17,18)$.

Entretanto, o envelhecimento por si só não diminui o interesse da mulher por sexo, nem seu potencial de reação sexual, especialmente se sua saúde geral for equilibrada. Ocorrem, porém, alterações fisiológicas específicas neste ciclo, após a menopausa, que não aparecem rapidamente, nem de maneira idêntica em todas (15,17-19,22,24-26).

Estas mudanças naturais durante o envelhecimento -como estreitamento, diminuição da elasticidade e das secreções vaginais, e desgaste de suas paredes-, favorecem o risco de infecção durante o sexo, sendo esta uma situação que, associada à percepção de risco, poderá conduzir mais idosas a adquirirem o HIV/AIDS $(15,22)$.

A tendência de crescimento do número de idosas na epidemia, sobretudo na subcategoria heterossexual, desde 1992, traduz-se na maior vulnerabilidade feminina e menor acesso aos serviços de saúde reprodutiva, pois não temem mais a gravidez indesejada e, consequentemente, não percebem a necessidade do uso de preservativo, que poderia prevenir a transmissão das DST; além disso, também é um fator importante a dificuldade em negociar o uso do preservativo com o parceiro $(4,5,7,9,15,22)$. 
Pesquisas demonstram que, em mulheres que convivem com HIV/AIDS, as marcas no corpo e a imagem a ser cuidada com o surgimento dos efeitos colaterais produzidos pelos medicamentos-são fatores coadjuvantes do conviver com o HIV/AIDS, que podem fortalecer o isolamento social e outros fatores negativos associados ao envelhecimento $(9,14,15,22,23)$.

Entretanto, há possibilidade de a idosa passar por um período de adaptação e descobertas do corpo feminino, apesar da doença, com necessidades sexuais possivelmente diferentes, porém não ausentes. Isso, porque a nudez geralmente traz vergonha, sensação de impotência, feiura, limitando-se a se expor apenas quando sentirse doente e dependente, fato que se agrava à idosa que convive com HIV/AIDS $(9,14-21)$.

Quando o idoso recebe o diagnóstico de infecção pelo HIV/ AIDS, impactos negativos nas atitudes e estereótipos relativos ao envelhecimento são potencializados, principalmente, em virtude das transformações advindas do envelhecimento. As modificações em nível dos componentes mentais têm sido utilizadas erroneamente como recurso para definir papéis e posições sociais hierárquicas, ou seja, muitos idosos passam a se comportar de maneira passiva, manipulados por outrem, desconfiando de sofrer de solidão e fortalecendo o ostracismo social, como já referido anteriormente (17-19,22,24-26).

Assim, utilizar de generalizações que levam a crer na progressiva e incompetência do idoso em exercer a sexualidade, faz parte destes estereótipos que culminam na dificuldade em preservar as identidades pessoais, sobretudo aquelas relativas à sexualidade, as quais a sociedade atentamente discrimina, principalmente quando se defende a possibilidade de sexualidade ativa, mesmo convivendo com HIV/AIDS $(9,14,15,23)$.

Sabe-se que a cultura brasileira repudia o interesse sexual de idosas, e ainda o considera, de maneira equivocada, como um arroubo da sexualidade, por uma juventude sexual mal vivida, tentando suprir a perda do tempo, estigmatizando a mulher e impedindo o desempenho da sua sexualidade $(9,17-19,21,24,25)$.

Diante disso, o desempenho da sexualidade por mulheres que convivem com o vírus praticamente se extingue, tendo em vista o preconceito, a desinformação, os valores socialmente determinados, 
e os valores religiosos, que implicam na culpa ou pecado, que, para elas, se apresentam como verdadeiros obstáculos, e impedem a retomada da sexualidade e de uma vida sem discriminação $(9,14,15,23)$.

Assim, o exercício da sexualidade precisa ser melhor compreendido, salientando que é possível ter uma relação sexual e exercer esta mesma sexualidade estando idoso, e ainda assim, conviver com HIV/AIDS. O preconceito social ao longo da história, que é o mesmo dos próprios idosos, precisa ser superado. A transformação da compreensão de sexualidade objetiva melhorar a autoestima e a visão que a idosa tem de si mesma, influindo, portanto, na feminilidade e, indiretamente, na sexualidade, alterando o seu papel social $(5,9,17-19,21)$.

Entretanto, é possível que a mulher idosa exerça a sedução e seja consciente da capacidade de despertar o desejo dos homens, além da sua capacidade sexual. Isso representa um amadurecimento na concepção de sexualidade, que gera consciência para atitudes espontâneas de desejos e vontades $(18,24)$.

No caso de viuvez e HIV/AIDS, a discriminação social é ainda maior, posto que reforça o agravamento de problemas mentais que podem levar ao suicídio, bem como o aparecimento de doenças como depressão e demência, que contribuem significativamente para a piora da condição de saúde, inclusive contribuindo para o comprometimento severo da imunidade já afetada $(9,14,15,22)$.

Entretanto, sempre há a possibilidade de recomeço, e uma das estratégias é o resgate da expressão da sexualidade, entendida como uma dimensão inerente à pessoa, presente em todos os atos de sua vida e determinante de um modo particular e individual de ser, existir, manifestar-se, comunicar-se, sentir e expressar, a qual tem a função de dar motivo à existência, para que estes indivíduos possam desempenhar atividades prazerosas a partir da sexualidade em sua plenitude e sem culpa, resultando, assim, na liberdade de conduzir suas vidas $(9,17-19,21,24,25)$. 


\section{Considerações finais}

Diante do exposto, deve-se considerar que os conflitos enfrentados pelos idosos, sobretudo aqueles que convivem com HIV/AIDS, merece atenção diferenciada e requer melhores discussões nas políticas públicas de saúde, já que a situação apresentada tende a agravar-se num curto espaço de tempo. Assim, compreendendo a complexidade que envolve a infecção, estratégias de planejamento e execução em saúde precisam ser implantadas e implementadas, dentre elas o trabalho com exercício da sexualidade, pois representa um princípio norteador de comportamentos sociais que exige muito cuidado.

Para isso, o estudo da bioética torna-se condição sin equa non para garantir a sexualidade aos idosos independente da idade, o que possibilita exercê-la de maneira responsável, a partir de normas morais e éticas sugeridas pelo trabalho em saúde pública, principalmente na atenção às populações especiais. Portanto, atributos desta população definidos historicamente pela sociedade, como as características de gênero, e acontecimentos como a feminização da velhice, devem ser considerados, confrontados e analisados, no intuito de ajudar a compor estratégias de cuidado melhor elaboradas que contemplem as necessidades dos idosos que convivem com HIV/AIDS. Afinal, a vida não se acaba no diagnóstico, mas recomeça na luta que se estabelece com o vírus a cada dia.

\section{Referências}

1. Bergman H, Ferrucci L, Guralnik J, Hogan DB, et al. An Emerging Research and Clinical Paradigm -Issues and Controversies. J Gerontol 2007; 62A (7): 731-737.

2. Kuh D. A Life Course Approach to Healthy Aging, Frailty, and Capability. J Gerontol 2007; 62A (7): 717-721.

3. IBGE. Instituto Brasileiro de Geografia e Estatística. Projeção da população do Brasil por sexo e idade 1980-2050 revisão 2008. Rio de Janeiro: IBGE; 2010.

4. Lazzarotto AR, Kramer AS, Hadrich M, Tonin M, et al. O conhecimento de HIV/AIDS na terceira idade: estudo epidemiológico no Vale do Sinos, Rio Grande do Sul, Brasil. Ciênc. saúde coletiva 2008; 13 ( 6 ): 1833-1840.

5. Pereira GS, Borges CI. Conhecimento sobre HIV/AIDS de Participantes 
de um Grupo de Idosos, em Anápolis-Goiás. Esc Anna Nery (impr.) 2010; 14 (4):720-725.

6. Araujo MAL, Araújo TL, Damasceno MMC. Conhecimento em HIV/ AIDS de 1998 a 2005: estudos publicados em periódicos de enfermagem. Esc Anna Nery 2006; 1(10): 126-31.

7. Brasil. Ministério da Saúde - Boletim Epidemiológico - AIDS e DST Ano VII - $\mathrm{n}^{\mathrm{o}} 1-27^{\mathrm{a}}$ a $52^{\mathrm{a}}$ - semanas epidemiológicas - julho a dezembro de 2009 - Ano VII - n 1 - 01 a 26 - semanas epidemiológicas - janeiro a junho de 2010.

8. Freitas EV. Tratado de geriatria e gerontologia. Rio de Janeiro: Guanabara Koogan; 2002.

9. Zornitta M. Os novos idosos com AIDS e desigualdades à luz da bioética (Dissertação de Mestrado). Rio de Janeiro (RJ): Escola Nacional de Saúde Pública Sérgio Arouca; 2008.

10. Costa S, Diniz D. Introdução a Bioética I. In: Costa SIF, Diniz D. Coordenadores. Bioética: ensaios. Brasilia: Letras Livres; 2001. p. 1316.

11. Beauchamp T, Childress JF. Principles of Biomedical Ethics. New York: Oxford University Press; 1979.

12. Schramm FR, Anjos MF, Zoboli ELCP. A questão das tendências epistemológicas ou de fundamentado. In: Anjos MF, Siqueira JE (Orgs.). Bioética no Brasil. Tendências e perspectivas. Aparecida (SP): Idéias\& Letras, São Paulo: Sociedade Brasileira de Bioética, 2007, p. 29-56.

13. Junges R J, Zoboli ELCP. Bioética e Saúde coletiva: Convergências Epistemológicas. Revista Ciência \& Saúde Coletiva para a sociedade 2012; 17(4): 1413-8123

14. Brasileiro M, Freitas FIM. Representantes Sociais sobre AIDS de pessoas acima de 50 anos de idade, infectadas pelo HIV. Rev LatAm Enfermagem 2006 set/out; 14(5) www.eerp.usp.br/rlae (Acesso em 5/ ago/2013).

15. Werba S, Alayde A, Araujo F, Ludgleydson, et al. Envelhecer com AIDS: Representantes, Crenças e Atitudes de Idosos Soropositivos para o HIV. Interam J Psychol 2009; 43(2):323-332.

16. Andrade HAS, Silva SK, Santos MIPO. AIDS em idosos: vivências dos doentes. Esc Anna Nery (impr.)2010; 14(4):712-719.

17. Almeida AL, Patriota LM. Sexualidade na terceira idade: um estudo com idosas usuárias do programa saúde da família do bairro das cidades Campina Grande/PB. Qualit@s Revista Eletrônica 2009; 8(1):1-20.

18. Negreiros MGT. Sexualidade e gênero no envelhecimento. ALCEU- jul./ dez. $2004 ;$ 5(9): 77- 86.

19. Catusso CM. Rompendo o silencio: desvelando a sexualidade em idosos. Revista Virtual Textos \& Contextos; $n^{\circ}$ 4, dez. 2005.

20. Garcia S, Koyama HAM. Estigma, discriminação e HIV/ Aids no contexto brasileiro, 1998 e 2005. Rev Saúde Pública 2008;42(Supl. 1):72-83. 
21. Vasconcellos D, Novo FR, Castro PO, DuryVK, et al. A sexualidade no processo do envelhecimento: novas perspectivas - comparação transcultural. Estudos de Psicologia 2004; 9(3): 413-419.

22. Araújo VLB, Brito DMS, Gimeniz MT, Queiroz TA et al . Características da Aids na terceira idade em um hospital de referência do Estado do Ceará, Brasil. Rev Bras Epidemiol 2007; 10(4): 544-54.

23. Garcia S, Koyama HAM. Estigma, discriminação e HIV/Aids no contexto brasileiro, 1998 e 2005. Rev Saúde Pública 2008;42(Supl1):72-83.

24. Fernandes MGM. Problematizando o corpo e a sexualidade de mulheres idosas: o olhar de gênero e geração. Rev. Enferm. UERJ, Rio de Janeiro, 2009 jul/set; 17(3):418-22.

25. Coelho PND, Daher VD, Santana FR, Santo EHF. Percepção de Mulheres Idosas sobre Sexualidade: implicações de gênero e no cuidado de enfermagem. Rev. Rene. Fortaleza, 2010 out/dez; 11(4): 163-173.

26. Souza THM, Backes SD, Pereira ADA, Ferreira LLC,et al. Nível de conhecimento de um grupo de idosos em relação à Síndrome da Imunodeficiência Adquirida. Avances em Enfermaría. VOL.XXVII. No 1. Enero-Junio 2009.

Recebido em: 01/06/2013 Aprovado em: 10/09/2013 